\title{
Resistivity in Cuprates and the Pseudogap
}

\author{
Sven Larsson ${ }^{1}$ (1)
}

Received: 7 June 2016 / Accepted: 11 October 2016 / Published online: 26 October 2016

(C) The Author(s) 2016. This article is published with open access at Springerlink.com

\begin{abstract}
The temperature dependence of resistivity $(\rho)$ in the normal state of $\mathrm{La}_{2-x} \mathrm{Sr}_{x} \mathrm{CuO}_{4}$ is calculated from electron transfer (ET) rates. The minimum at about $100 \mathrm{~K}$ for doping levels less than $6 \%$ is characteristic for all cuprates. The resistivity depends on ET between $\mathrm{Cu}$ (III) and $\mathrm{Cu}$ (II) sites. For $T<300 \mathrm{~K}$, the resistivity is proportional to $T^{3 / 2}$. The more than linear increase of $\rho$ for $T>100 \mathrm{~K}$ depends on increased kinetic energy in a Landau avoided crossing. The agreement with experimental data is convincing. The behaviour for $T \rightarrow 0$ can be explained as nuclear tunnelling. For $T>400 \mathrm{~K}, \rho$ bends down, presumably because the pseudogap is overcome at a higher kinetic energy.
\end{abstract}

Keywords Cuprate - Resistivity minimum .

Superconducting gap · Pseudogap · Landau-Pekar model . Marcus model $\cdot$ Jortner model $\cdot$ Nuclear tunnelling

\section{Introduction}

High- $T_{\mathrm{C}}$ superconductors are said to be strongly correlated systems which, in principle, means that a mean field approach for electron motion is impossible as a description. A better alternative would be to use the free energy models of Marcus or Jortner for electron transfer or electron pair transfer [1-3] and derive an expression for resistivity based on electron transfer (ET) rates. Such an approach involves

Sven Larsson

slarsson@chalmers.se

1 Department of Chemistry, Chalmers University of Technology, Old Physics Building, S-412 96 Gothenburg, Sweden a calculation of the probability for site-to-site transfer, based on spectroscopic information or quantum chemical calculation $[4,5]$.

Let us consider two adjacent copper sites, A and B, in a cuprate crystal. As a function of collective nuclear configuration coordinates (for example, along a breathing vibration), the free energy is rising parabolically from two possible equilibrium configurations: one for a state with equal charges on the copper sites $[\mathrm{Cu}(\mathrm{II})+\mathrm{Cu}(\mathrm{II})]$ in the ground state of the cuprates (in the normal region) and one for an excited state with disproportionated charges $[\mathrm{Cu}(\mathrm{I})+\mathrm{Cu}(\mathrm{III})]$. The interactions between the two states lead to avoided crossings. The interdependence of electronic density and nuclear positions is mentioned as the "internal contradiction" in a paper by Landau and Pekar [6]. These authors propose to use effective mass in a theory which resembles the modern quasiparticle approach. However, the dependence of structure on the number of electrons in a local system is still ignored in most papers. In fact, the quasiparticle approach has not been very successful in explaining high- $T_{\mathrm{C}}$ superconductivity (SC).

In the Marcus and Jortner theories used here, the dependence on nuclear coordinates is taken into account. For example in $\mathrm{Cu}(\mathrm{I}), \mathrm{Cu}(\mathrm{II})$ and $\mathrm{Cu}(\mathrm{III})$ systems, the relevant $\mathrm{CuO}$ bond length is reduced by about $0.1 \AA$ for each new electron removed. This is due to charge on copper and the fact that the electrons removed belong to antibonding orbitals.

The Marcus and Jortner models are here extended to a theory for electron mobility from which the conductivity is obtained [5]. According to the model, resistivity in the normal state in a cuprate has a minimum for $T=\left(2 E_{\mathrm{a}}\right) /\left(3 k_{\mathrm{B}}\right)$, where $E_{\mathrm{a}}$ is the activation energy [7,8]. For slightly doped $\mathrm{La}_{(2-x)} \mathrm{Sr}_{x} \mathrm{CuO}_{4}(x<0.06), T_{\min } \approx 100 \mathrm{~K}$ and, therefore, the activation energy $\left(E_{\mathrm{a}}\right)=0.035 \mathrm{eV}$, which agrees 
with Hall effect data and the results of Takagi et al. for the resistivity [9].

Spectral data obtained by Uchida et al. [10] have been used. The absorption peak at $2 \mathrm{eV}$ (Hubbard $U$ ) in the photo-induced conductivity spectra of $\mathrm{La}_{2} \mathrm{CuO}_{4}$ has not been correctly assigned in the literature [7]. It is due to the $\mathrm{Cu}-\mathrm{Cu}$ charge transfer $(\mathrm{CT})[7,8]$, not to the $\mathrm{O} 2 p \rightarrow \mathrm{Cu} 3 d$ CT. If the relevant negative terms are included, $U$ is not always $>0$.

The results obtained for the resistivity as a function of temperature agree well with the experimental results [9]. For $T \rightarrow 0$, the Jortner model [3], which includes nuclear tunnelling, is used.

Usually, a larger charge (fewer electrons) of the metal ion at the site leads to higher self-trapping probability. The "optical anomaly" consisting of transfer of spectral weight when the superconducting state is approached [10] is directly related to doping and creation of $\mathrm{Cu}$ (III) sites. $U$ depends on dopant concentration.

Finally, the pseudogap is discussed in the light of the results of the simulation. The disproportionation reaction

$2 \mathrm{Cu}(\mathrm{II}) \rightarrow \mathrm{Cu}(\mathrm{I})+\mathrm{Cu}(\mathrm{III})$

is the origin of both the SC gap and the pseudogap [8].

\section{Charge Transfer, Gap and Pseudogap}

The maximum of photo-induced conductivity at $2 \mathrm{eV} \mathrm{[10]} \mathrm{is}$ often ascribed to ligand-to-metal (LM)CT (O2p $\rightarrow \mathrm{Cu} 3 d)$. It is hard to see that LMCT can lead to conductivity, and there is no proof for it. LMCT is, in fact, related to the term "CT insulators", since the LMCT excitation is always within the site of a metal ion and its closest ligands and therefore cannot lead to conductivity.

On the other hand, transitions between two metal sites lead to photo-induced conductivity. Hubbard $U$ is the metalto-metal charge transfer (MMCT) transition [11]. Cuprates and $\mathrm{CuO}$ are brown or black due to a strong absorption at 1.5-2.5 eV. MMCT transitions are charge separation processes and therefore strongly depend on the distance for electron transfer. Increased distance for electron transfer costs more in energy. In aqueous solutions of $\mathrm{Cu}$ (II), the $\mathrm{CT}$ distance is larger than that in a cuprate and, therefore, the MMCT transition appears in the UV region. The blue colour of $\mathrm{CuSO}_{4}$ is due to ligand field transitions. LMCT transitions, on the other hand, are located well above the ligand field transitions, and there is no distance dependence.

The active orbitals, forming the SC gap and the pseudogap, are of the type $\mathrm{Cu} 3 d\left(x^{2}-y^{2}\right)$ [mixed with $\mathrm{O} 2 p(x)$ and $\mathrm{O} 2 p(y)]$. If the orbital is called $a$ on site $\mathrm{A}$ and $b$ on site $\mathrm{B}$, the ground state may be written as $a b+b a$, forming the spin-coupled ground state of the undoped cuprates. $a a+b b$ and $a a-b b$ are the charged states at $1.7-2.5 \mathrm{eV}$. The adiabatic process, where the nuclear positions adopt to the different occupancies, is at a much lower energy.

This picture is correlated but is, nevertheless, comparable to the accepted picture, in a review by Damascelli et al. [12, Fig. 13]. The upper Hubbard band in a cuprate corresponds to the $a a+b b$ and $a a-b b$ states and the lower Hubbard band to $a b+b a$. The energy difference is vertical Hubbard transition, $U_{\text {vert }}$. The orbitals of the local picture begin to out-localize themselves on the neighbours, on the way to become infinitely extended. The almost forbidden ligand field transitions, on the other hand, are hidden below the MMCT (Hubbard) transitions.

After sufficient doping, Hubbard $U$ tends to zero. The ground state in the superconductor is a superposition of $a b+b a$ and $a a+b b[7,8]$.

Among the active orbital states, there are two possible transitions: from the symmetric ground state to the $a a-b b$ state and from the $a a-b b$ state to the symmetric excited state when $a a-b b$ becomes thermally occupied. The former transition constitutes the gap and the latter (very likely) the pseudogap.

The low energy for MMCT in the cuprates (Hubbard $\left.U_{\text {vert }}\right)$ is consistent with the fact that copper has three stable oxidation states: $\mathrm{Cu}(\mathrm{I}), \mathrm{Cu}(\mathrm{II})$ and $\mathrm{Cu}$ (III). The nature of the MMCT transition at $2 \mathrm{eV}$ follows from the polarization in the $\mathrm{CuO}_{2}$ plane ( $x, y$ plane). The transition moment may be written as

$M=\int(a a-b b) e\left(x_{1}+x_{2}\right)(a b+b a) d x d y d z=2 R$

where $R$ is the distance between the sites and $e$ is the electron charge. The same result is obtained for the $y$ coordinates while the $z$-coordinates give no contribution to $M$. The polarization vector is thus in the plane.

Mott included on-site electron repulsion but neglected attractive ionic terms [11]. $U \approx 10-20 \mathrm{eV}$ if defined that way. In cuprates, $U$ is measured spectroscopically as $2 \mathrm{eV}$ before doping, as a Franck-Condon transition. If the nuclear positions are allowed to vary to minimize the energy, we obtain two equilibria separated by adiabatic $\left(U_{\mathrm{ad}}\right)$. $U_{\text {ad }} \ll$ $U_{\text {vert }}$ and only $U_{\text {ad }}$ has to become $\leq 0$ as a condition for SC.

The transfer of spectral density as the doping proceeds in, for example, $\mathrm{La}_{2-x} \mathrm{Sr}_{x} \mathrm{CuO}_{4}$ [10] and can be explained [7]. In $\mathrm{La}_{2} \mathrm{CuO}_{4}$, the MMCT transition at $2 \mathrm{eV}$ may be written as

$h v_{1}+\mathrm{Cu}_{\mathrm{A}}(\mathrm{II})+\mathrm{Cu}_{\mathrm{B}}(\mathrm{II}) \rightarrow \mathrm{Cu}_{\mathrm{A}}(\mathrm{I})+\mathrm{Cu}_{\mathrm{B}}(\mathrm{III})$

Doping creates holes in the form of $\mathrm{Cu}$ (III) sites. MMCT into these holes certainly has a lower energy than if transferred to a $\mathrm{Cu}(\mathrm{II})$ site. When $\mathrm{Cu}(\mathrm{III})$ sites are being 
created at increased doping, another excitation mechanism takes over

$h v_{2}+\mathrm{Cu}_{\mathrm{A}}(\mathrm{II})+\mathrm{Cu}_{\mathrm{B}}(\mathrm{III}) \rightarrow \mathrm{Cu}_{\mathrm{A}}(\mathrm{III})+\mathrm{Cu}_{\mathrm{B}}(\mathrm{II})$

where $h v_{2}$ is the vertical transition of Fig. $1(\lambda)$ and obviously $v_{2}<v_{1}$.

At higher doping levels, the holes are closer to each other and the energy decreases further. In the spectrum of Uchida et al. [10] of $\mathrm{La}_{2-x} \mathrm{Sr}_{x} \mathrm{CuO}_{4}$, the hardly visible absorption at $0.4 \mathrm{eV}$ is increasing in intensity and decreasing in energy for $x>0$. The increase in intensity up to $1 \mathrm{eV}$ is roughly proportional to the doping, and this determines when (4) becomes important for the optical conductivity and (5) for the thermal conductivity. The Drude peak is much increased in intensity. All cuprates show this absorption in the near-infrared (NIR) spectra, and the reason seems to be the intersection between the $\mathrm{Cu}(\mathrm{II})+\mathrm{Cu}$ (II) and $\mathrm{Cu}(\mathrm{I})+\mathrm{Cu}(\mathrm{III})$ energy surfaces $[7,8]$.

\section{Electron Mobility due to Single Electron Transfer}

If the left minimum in Fig. 1 corresponds to the system $\mathrm{A}^{+} \mathrm{B}$, the right minimum corresponds to the system $\mathrm{AB}^{+}$. The coupling between electronic and nuclear motions lies in the definition of the reaction coordinate [11]. In a transition metal system, the highest occupied orbitals are antibonding orbitals. Hence occupying such an orbital, the bond lengths are increased and the charge of the metal ion is decreased. Thus, the site with the largest number of electrons has the largest bond lengths.

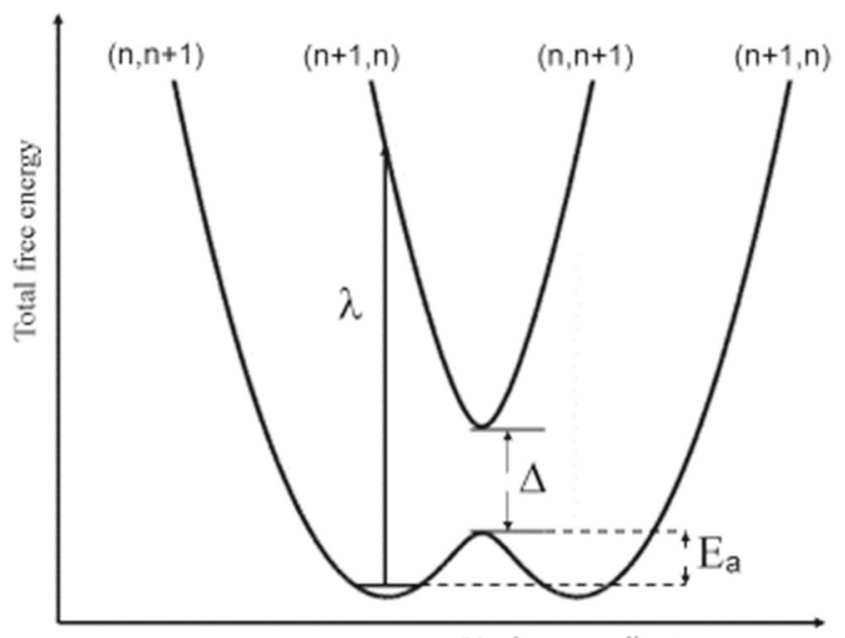

Nuclear coordinate

Fig. 1 Potential free energy surfaces in the Marcus model for one electron transfer. $\lambda$ is the reorganization energy, $\Delta=2 H_{12}$ the coupling and $E_{\mathrm{a}}$ the activation energy. $(n, n+1)$, etc., refers to site orbital occupation
We first consider the single electron transfer case

$\mathrm{Cu}_{\mathrm{A}}(\mathrm{II})+\mathrm{Cu}_{\mathrm{B}}(\mathrm{III}) \rightarrow \mathrm{Cu}_{\mathrm{A}}(\mathrm{III})+\mathrm{Cu}_{\mathrm{B}}(\mathrm{II})$

where A and B are adjacent sites. The barrier between the minima in Fig. $1\left(E_{\mathrm{a}}\right)$ has to be passed. The rate constant is proportional to $\exp \left(-E_{\mathrm{a}} / k_{\mathrm{B}} T\right)$. The barrier arises when a single electron or hole passes from one site to the next and the bond lengths change. The system is then passing from one minimum to the other in Fig. 1. We notice that without coupling, $(\Delta=0) E_{\mathrm{a}}=\lambda / 4$. If $|\Delta|$ is large enough, the barrier will disappear. If the barrier disappears, the system is delocalized.

The energy gap $(\Delta)$ is due to the electronic interaction between the two states of the energy surfaces. If $\Delta$ is very small, the presence of the other energy parabola will pass unnoticed, and the electron remains in its site. $\lambda=U_{\text {vert }}$, i.e. the barrier height, subtracted by the zeropoint vibrational energy, is equal to the activation energy $\left(E_{\mathrm{a}}\right)$.

The electron mobility is derived as a diffusion problem $[5,8]$ where the probability in each step is the reaction rate $(k)$ in the Marcus or Jortner models [1-3].

Figure 1 is independent of doping since the overwhelming majority of leaps are to the neighbouring copper site. $\lambda=U_{\text {vert }}$ is the vertical excitation energy and $U_{\text {ad }}$ the energy difference between the minima.

The Drude model may be used to calculate the conductivity in an infinite ET system, provided that the electrons do not interact with each other as in a real metal. The difference between forward and backward ETs is calculated in an infinitesimal field. The current is the product of carrier mobility and carrier concentration $(n)$ in the case $\nu_{\mathrm{n}} \gg v_{\mathrm{el}}$

$\sigma=n \cdot$ const. $\cdot \frac{\Delta^{2}}{4 \pi \hbar}\left(\frac{\pi^{3}}{\lambda k_{\mathrm{B}}}\right)^{1 / 2} \cdot\left(\frac{1}{T}\right)^{3 / 2} \exp \left(-\frac{\lambda}{4 k_{\mathrm{B}} T}\right)$

The activation energy is $\lambda / 4$, and the resistivity $(\rho=1 / \sigma)$

$\rho=$ const. $^{-1} \cdot \frac{1}{n} \cdot \frac{4 \pi \hbar}{\Delta^{2}} \cdot\left(\frac{\lambda k_{\mathrm{B}}}{\pi^{3}}\right) \cdot T^{3 / 2} \exp \left(\frac{\lambda}{4 k_{\mathrm{B}} T}\right)$

In the low-doping region, we may associate the carrier concentration with the molar fraction of $\mathrm{Cu}$ (III) carriers, thus $n \sim p=x$.

$E_{\mathrm{a}}$ may be calculated as

$E_{\mathrm{a}}=\frac{\lambda}{4} \cdot\left(1-\frac{\Delta}{\lambda}\right)^{2}$

$E_{\mathrm{a}}=\lambda / 4$ for $\Delta=0$ and $E_{\mathrm{a}}=0$ for $\lambda=\Delta$. If $|\Delta| \ll \lambda$, the activation energy depends only on $\lambda$ and is equal to $\lambda / 4$.

If $|\Delta|=\lambda$, on the other hand, the barrier disappears $\left(E_{\mathrm{a}}=0\right)$ :

$\rho=v_{\mathrm{n}}^{-1}$ const. $^{-1} \cdot \frac{1}{p} \cdot T$ 
Equation (9) holds only in the case of very strong coupling. $\rho$ is then proportional to $T$ (Einstein case). In the Einstein case, the power on $1 / T$ disappears in (6) (the electrons are always hopping if the barrier is overcome). This is the case in (9). If the particles move fast, on the other hand, there is a slowdown originating from the Landau probability for hopping rate. The electrons have to "collect probability" for the hopping event. Therefore, there is an additional factor $T^{-1 / 2}$ in (6).

Oxidative doping of a cuprate leads to a $\mathrm{Cu}(\mathrm{II}) / \mathrm{Cu}$ (III) mixed-valence system and possible exchange of electrons and hence conductivity. The conductivity is activated like in semiconductors, but due to structural relaxation rather than a band gap. We may calculate a minimum $\left(\rho_{\min }\right)$ in

$\rho=T^{m} \exp \left(\frac{E_{\mathrm{a}}}{k_{\mathrm{B}} T}\right)$

for $T_{\min }=\frac{1}{m} \cdot \frac{E_{\mathrm{a}}}{k_{\mathrm{B}}}$

where $m$ is the power of $T$ in the expression for the conductivity. $m=3 / 2$ in the present case if the Marcus model holds, as in (7). In the case of semiconductors, $m=0$, and hence, there is no minimum. In the plain Einstein case [13], $m=1$, and in the case of (7) above, $m=3 / 2$. In the case of electron pair transfer, there are two successive Landau-Zener avoided crossings (Fig. 3) and hence $m=2$. The existence of a minimum in the results of Takagi et al. [9] is an ample proof that the activation is not due to a semiconductor gap.
Activation due to structural relaxation $[m=3 / 2$ (rather than $m=0$ ) in (11)] leads to a minimum in the resistivity as a function of temperature. The simulation based on Eqs. (5) and (7) is shown in Fig. 2. The visible agreement with the results of Takagi et al. [9] below $400 \mathrm{~K}$ suggests that one-electron exchange is in operation.

The more than linear raise in resistivity for higher temperatures is due to larger kinetic energy through the LandauZener crossing. For low temperatures, the classical Marcus model has to be replaced by the Jortner model, due to nuclear tunnelling.

In Fig. 2, the activation energy is $0.035 \mathrm{eV}$ [7], which corresponds to $\mathrm{Cu}(\mathrm{III}) / \mathrm{Cu}(\mathrm{II})$ exchange, since in agreement with the Marcus model, there is a photo-induced conductivity peak at $4 \times 0.035=0.014 \mathrm{eV}$ [14]. The coupling parameter between two $\mathrm{Cu}$ ions, through an oxygen ion, has quite arbitrarily been chosen as $H_{12}=0.0065 \mathrm{eV}$ which gives the gap $\Delta=0.013 \mathrm{eV}$. We obtain Fig. 2. Incidentally, the activation energy for the Hall factor is also $0.035 \mathrm{eV}$ [15].

Above $T=400 \mathrm{~K}$, the resistivity bends down [9], signalling a novel conductivity mechanism. This bending down for higher temperatures seems to be caused by the pseudogap [8]. The activation energy for thermal excitation is hard to approximate, since it depends on both $U_{\mathrm{ad}}$ and the coupling. If it is set to $0.175 \mathrm{eV}$, there is good agreement with the experiments [8].

Increasing the doping above $x>0.05$ leads to SC (Fig. 2, right figure). As discussed in papers by the present author $[16,17]$, this depends on the increased doping level which
Fig. 2 Left figure: calculated resistivity for $\mathrm{La}_{2-x} \mathrm{Sr}_{x} \mathrm{CuO}_{4}$. Right figure: experimental results, taken from ref. [9]

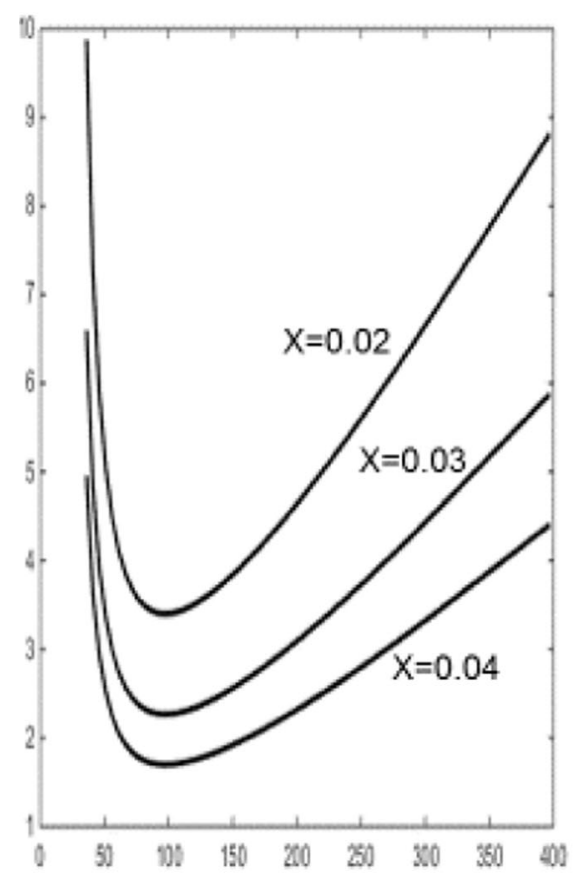


puts the spin-coupled and charged states on equal energy, or with $U_{\text {ad }}$ slightly smaller than zero. If there is strong interaction between these states and with a half-breathing vibrational mode, the resulting states have a SC gap. The ground state is SC, and the higher state, the so-called Bogoliubov state, prevents SC. When excitations across the gap become thermally possible, SC disappears.

\section{Resistivity for $T \rightarrow 0$}

The calculated results of Fig. 2 do not agree with the experimental ones for low temperatures. In the Marcus model, the nuclei move by classical mechanics. If the temperature is low so that $k_{\mathrm{B}} T$ is smaller than the vibrational energies, the classical model does not behave satisfactorily and the nuclear vibrations have to be included in quantized form. In chemical physics, this is done using the Jortner model [3]. The logarithm of the conductivity tends to linearly decrease as a function of $1 / T$ [4]. The Jortner model includes "nuclear tunnelling" which means a slower decrease than linear for low temperatures [3]. The resistivity tends to have finite values as $T \rightarrow 0$ in the Jortner model, and this agrees with experimental observation [18]. In the "variable range hopping" model, on the other hand, the resistivity tends to infinity as $1 / T^{1 / 4}$ as $T$ tends to zero, against experimental trends [18].

\section{Electron Pair Mobility}

In the electron pair transfer problem, the two energy parabolas are energies for states with an even number of electrons (Fig. 3). In such a state, there are two additional electrons, called active electrons, on one of the sites. The direct coupling between states where both electrons are on either one or the other site ( $a a$ and $b b$ below) is very small. The main coupling is indirect via the spin-coupled state $(a b+b a$ below). We thus introduce the parabola corresponding to the spin-coupled state with one active electron on each site (Fig. 3).

We assume two sites. On the $x$-axis of Fig. 3, there are plotted collective nuclear coordinates for a breathing or half-breathing motion. In other words, when the ligand coordinates around one site shrink, they open up on the other site. The active electrons are highly sensitive to the nuclear positions and localize in regions with the lowest energy.

The leftmost minimum in total free energy is the equilibrium point if there are two additional electrons on one of the metal ions. If there was no interaction with the other metal site, the energy curves may be approximated as perfect parabolas, meaning that the electrons would remain on one of the sites for ever. When we move to the right on the $x$-axis, we will reach the minimum for the $\mathrm{Cu}$ (II)/Cu(II) state, corresponding to the spin-coupled ground-state wave function with one electron on each site.

$\mathrm{SC}$ requires that $a a+b b$ is the major part of the groundstate wave function, since the superconducting gap is between $a a+b b$ and $a a-b b$. Thus, $U_{\mathrm{ad}} \leq 0$ is a condition for SC. Another condition is that the coupling is so large that the barrier disappears. If $U_{\text {ad }}$ is very negative, this cannot happen and the system will be locked in the charged state. In pure $\mathrm{La}_{2} \mathrm{CuO}_{4}$, the ground state is dominated by the spincoupled state $\left(U_{\mathrm{ad}}>0\right)$ represented by the right parabola.

In the spectra of Uchida et al. [10], there is photo-induced conductivity between 0 and $1 \mathrm{eV}$ already at $2 \%$ doping, with a maximum of $0.6 \mathrm{eV}$. The constant background, probably
Fig. 3 Potential energy surfaces (PESs) for electron pair transfer according to the Marcus model

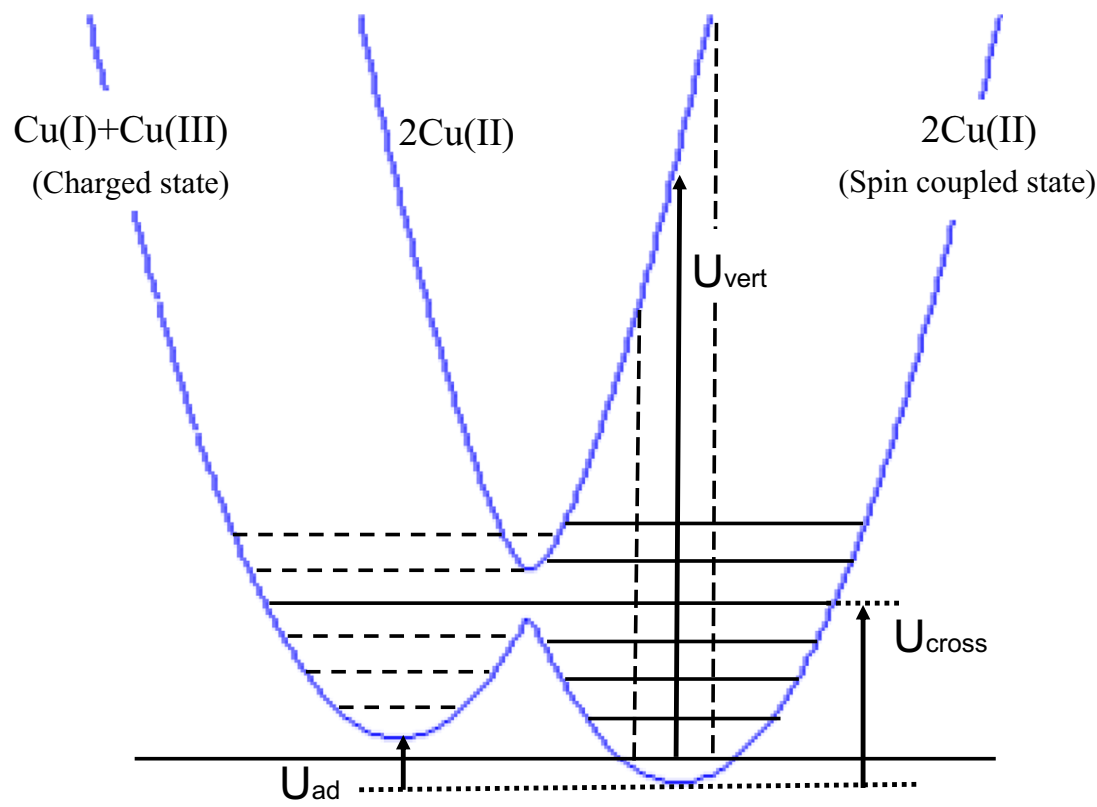


due to one electron transfer, is not changing very much for higher doping, but the maxima increase in size and move to lower energies, before it goes over to Drude absorption. It is believed [8] that this is the pseudogap $(a b+b a \rightarrow a a-b b)$.

\section{Conclusion}

The results obtained are encouraging and confirm that SC appears for Hubbard $U \leq 0$, provided that some other conditions are satisfied, for example that the reaction barriers disappear in Fig. 3, which is favoured by the equal free energy between spin-coupled and charged states and the strong interaction between the metal sites.

An important observation is that the activation energy for one electron transport is to a large extent independent of doping. The states induced by one electron transfer are due to single electrons moving between two adjacent sites. As for $\mathrm{CuO}$, there always remains activation energy. This means that $\mathrm{Cu}(\mathrm{III})$ is, in fact, localized, quite consistent with the appearance of stripes [19].

SC in the cuprates appears after $5 \%$ doping. We interpret that there is equal energy for the spin-coupled and charged sites $\left(U_{\mathrm{ad}}=0\right)$ and, therefore, there is quantum mechanical configuration interaction forming a new SC phase. The SC gap and the pseudogap are formed, always involving two electrons. This means that the Bose-Einstein statistics becomes "visible", for example, in the heat capacity.

Open Access This article is distributed under the terms of the Creative Commons Attribution 4.0 International License (http:// creativecommons.org/licenses/by/4.0/), which permits unrestricted use, distribution, and reproduction in any medium, provided you give appropriate credit to the original author(s) and the source, provide a link to the Creative Commons license, and indicate if changes were made.

\section{References}

1. Marcus, R.A.: Ann. Rev. Phys. Chem. 15, 155 (1964)

2. Brunschwig, B.S., Logan, J., Newton, M.D., Sutin, N.: J. Am. Chem. Soc. 102, 5798-5809 (1980)

3. Jortner, J.: J. Chem. Phys. 64, 4860-4867 (1976)

4. Larsson, S.: Int. J. Quant. Chem. 30, 31-49 (1986)

5. Johansson, E., Larsson, S.: Synth. Met. 144, 183-191 (2004)

6. Landau, L.D., Pekar, S.I., Eksp, Zh.: Teor. Fiz. 18, 419-423 (1948). (in Russian). English translation: Ukr. J. Phys., Special issue 53, 71-74 (2008)

7. Larsson, S.: Appl. Phys. Res. 7, 14-23 (2015)

8. Larsson, S.: J. Supercond. Nov. Magn. (2016). doi:10.1007/ s10948-016-3666-0

9. Takagi, H., Batlogg, B., Kao, H.L., Kwo, J., Cava, R.J., Krajewski, J.J., Peck, W.F. Jr.: Phys. Rev. Lett. 69, 2975-2978 (1992)

10. Uchida, S., Ido, T., Takagi, H., Arima, T., Tokura, Y., Tajima, S.: Phys. Rev. B 43, 7942-7954 (1991)

11. Mott, N.F.: Revs. Mod. Phys. 40, 677-683 (1968)

12. Damascelli, A., Hussain, Z., Shen, Z.-X.: Revs. Mod. Phys. 75, $473(2003)$

13. Einstein, A.: Annalen der Physik 322, 549-560 (1905)

14. Falck, J.P., Levy, A., Kastner, M.A., Birgeneau, R.J.: Phys. Rev. B 48, 4043-4046 (1993)

15. Ando, Y., Komiya, S., Segawa, K., Ono, S., Kurita, Y.: Phys. Rev. Lett. 93, 267001(4) (2004)

16. Larsson, S.: Chem. Phys. 236, 133-150 (1998)

17. Larsson, S.: J. Mod. Phys. 4, 29-32 (2013)

18. Ando, Y., Boebinger, G.S., Passner, A., Wang, N.L., Geibel, C., Steglich, F.: Phys. Rev. Lett. 77, 2065-2068 (1996)

19. Tranquada, J.M., Sternlieb, B.J., Axe, J.D., Nakamura, Y., Uchida, S.: Nature 375, 561-563 (1995) 